\title{
Distinct features of rabbit and human adipose- derived mesenchymal stem cells: implications for biotechnology and translational research
}

This article was published in the following Dove Press journal: Stem Cells and Cloning:Advances and Applications

\author{
Helena Debiazi Zomer ${ }^{1,2}$ \\ Kelly CS Roballo ${ }^{2}$ \\ Thais Borges Lessa ${ }^{2}$ \\ Fabiana Fernandes Bressan ${ }^{2}$ \\ Natália Nardeli Gonçalves ${ }^{2}$ \\ Flávio Vieira Meirelles ${ }^{2,3}$ \\ Andrea Gonçalves Trentin' \\ Carlos Eduardo Ambrósio 2,3 \\ 'Department of Cell Biology, \\ Embryology and Genetic, Faculty \\ of Biological Sciences, Santa \\ Catarina Federal University (UFSC), \\ Florianópolis, Brazil; ${ }^{2}$ Department \\ of Surgery, Sector Anatomy, Faculty \\ of Veterinary Medicine and Animal \\ Science, University of São Paulo, \\ São Paulo, Brazil; ' ${ }^{3}$ Department of \\ Veterinary Medicine, Faculty of \\ Animal Science and Food Engineering, \\ University of São Paulo, São Paulo, \\ Brazil
}

Correspondence: Carlos Eduardo Ambrósio

Department of Veterinary Medicine, Faculty of Animal Science and Food Engineering, University of São Paulo, Avenida Duque de Caxias Norte, 225, Pirassununga, São Paulo 13635-900, Brazil Tel +55 I| 35654 I I2

Email ceambrosio@usp.br
Introduction: Owing to their similarity with humans, rabbits are useful for multiple applications in biotechnology and translational research from basic to preclinical studies. In this sense, mesenchymal stem cells (MSCs) are known for their therapeutic potential and promising future in regenerative medicine. As many studies have been using rabbit adipose-derived MSCs (ASCs) as a model of human ASCs (hASCs), it is fundamental to compare their characteristics and understand how distinct features could affect the translation to human medicine.

Objective: The aim of this study was to comparatively characterize rabbit ASCs (rASCs) and hASCs to further uses in biotechnology and translational studies.

Materials and methods: rASCs and hASCs were isolated and characterized by their immunophenotype, differentiation potential, proliferative profile, and nuclear stability in vitro.

Results and discussion: Both ASCs presented differentiation potential to osteocytes, chondrocytes, and adipocytes and shared similar immunophenotype expression to CD105+, CD34-, and CD45-, but rabbit cells expressed significantly lower CD73 and CD90 than human cells. In addition, rASCs presented greater clonogenic potential and proliferation rate than hASCs but no difference in nuclear alterations.

Conclusion: The distinct features of rASCs and hASCs can positively or negatively affect their use for different applications in biotechnology (such as cell reprogramming) and translational studies (such as cell transplantation, tissue engineering, and pharmacokinetics). Nevertheless, the particularities between rabbit and human MSCs should not prevent rabbit use in preclinical models, but care should be taken to interpret results and properly translate animal findings to medicine.

Keywords: characterization, comparison, MSC, iPS, immunophenotype, proliferation

\section{Introduction}

Rabbits (Oryctolagus cuniculus) are widely used as experimental models for both human and veterinary medicine by their ease to work with and relatively economic maintenance. ${ }^{1}$ Their size is ideal for surgical procedures and allow for the use of a high number of animals in an experiment, improving statistical relevance. ${ }^{2,3}$ Owing to their similarity with humans, rabbits are useful for multiple applications in biotechnology and translational medicine from basic research to preclinical studies, such as fertilization in vitro, embryonic development and organogenesis, immunology, toxicology, neurophysiology, ophthalmology, and cardiology. ${ }^{4}$

In this sense, translational studies have been testing the efficacy of mesenchymal stem cells (MSCs) to treat diverse human diseases and conditions..$^{5-7}$ The MSCs are 
characterized by their potential to generate different mesodermal tissues and auto-renew. ${ }^{8}$ In the body, MSCs contribute to postnatal development by the substitution of dead cells..$^{9,10}$ When transplanted, they exert therapeutic effects by the direct differentiation in various cell types and paracrine function by the secretion of cytokines and growth factors. ${ }^{11}$

The adipose tissue is an important source of MSCs highlighted by its accessibility, safety to collect, and abundance of cells. ${ }^{12-14}$ The therapeutic potential of adipose-derived MSCs (ASCs) was proved in different contexts, such as skin wound healing, bone fractures, and cancer immunosuppression. ${ }^{12,15,16}$ Thus, ASCs present promising potential to future regenerative medicine approaches by their anti-inflammatory properties, differentiation potential, angiogenesis induction, and paracrine effects.

The ASCs were previously isolated from various animals, including humans. ${ }^{17,18}$ Although sharing basic characteristics, MSCs from different sources and species present individual features, with possible consequences to future therapeutic applications. As many studies have been using rabbit ASCs (rASCs) as a model of human ASCs (hASCs), it is fundamental to compare their characteristics and understand how their distinct features could affect the translation to human medicine. Therefore, the aim of this study was to comparatively characterize rASCs and hASCs to further uses in biotechnology and translational studies.

\section{Materials and methods}

\section{Ethics committee approval}

This study was approved by the research ethics committee of Faculty of Veterinary Medicine and Animal Science, University of São Paulo, Brazil (2560/2012) and by the ethics committee for human research of Santa Catarina Federal University, Brazil (1.076.626).

\section{Isolation of adipose-derived MSCs}

The rASCs were obtained from New Zealand rabbits $(n=6)$ at the slaughterhouse of University of São Paulo (Pirassununga,
Brazil). The hASCs were isolated from human subcutaneous fat samples $(n=6)$ discarded from abdominoplasties at the Hospital of Santa Catarina Federal University (Florianopolis, Brazil) after donors' written informed consent. The same isolation protocol was used for rASC and hASC obtainment.

Briefly, the samples ( $3 \mathrm{~g})$ were washed with PBS supplemented with $1 \%$ of penicillin and streptomycin (Thermo Fisher Scientific, Waltham, MA, USA) and transferred to Petri dishes with $5 \mathrm{~mL}$ of collagenase IV $0.4 \%$ (SigmaAldrich Co., St Louis, MO, USA). Adipose tissue fragments were minced with scalpels for 5 minutes and incubated at $37^{\circ} \mathrm{C}$ for $10-20$ minutes. Samples were then centrifuged at $300 \times g$ for 5 minutes, and the supernatants were discarded and cell pellets suspended in the complete culture medium composed of DMEM/F12 (LGC Biotecnologia, Cotia, Brazil) supplemented with $10 \%$ fetal bovine serum (Vitrocell, Campinas, Brazil), 1\% l-glutamine (Sigma-Aldrich Co.), and $0.5 \%$ penicillin and streptomycin.

\section{Flow cytometry}

Flow cytometry was performed in second passage rASCs and hASCs for detection of surface antigenic markers CD34, CD45, CD73, CD90, and CD105 (BD Bioscience, São Paulo, Brazil; Table 1). White blood cells fractions were used as positive controls for CD34 and CD45. Negative control staining was performed by using fluorophore-conjugated mouse IgG isotype antibodies. Briefly, cell suspensions were distributed into $15 \mathrm{~mL}$ conic tubes, washed twice with PBS, and fixed in paraformaldehyde (4\%) for 15 minutes at room temperature. After two more washes with PBS, cells were centrifuged at $500 \times g$ for 5 minutes and incubated with blocking buffer composed of PBS+2\% BSA (Sigma-Aldrich Co.) at room temperature for 45 minutes. Cells were centrifuged at $500 \times g$ for 5 minutes, washed twice with buffer composed of PBS+BSA $(0.2 \%)$, and incubated with the antibodies solution (1:100) for 1 hour at $4^{\circ} \mathrm{C}$ in the absence of light. Samples were washed, and pellets were suspended in paraformaldehyde $4 \%$ for flow cytometry analysis (FACSCalibur; BD Biosciences,

Table I Antibodies information

\begin{tabular}{|l|l|l|l|l|}
\hline Antibody & Isotype & Reacts with & $\begin{array}{l}\text { Conjugated } \\
\text { fluorophore }\end{array}$ & $\begin{array}{l}\text { Brand and catalog } \\
\text { number }\end{array}$ \\
\hline Anti-CD34 & Mouse lgGI & Human & PE & BD Pharmingen, 555822 \\
Anti-CD45 & Mouse lgGI & Human & FITC & BD Pharmingen, 555482 \\
Anti-CD73 & Mouse lgGI & Human & PE Pharmingen, 550257 \\
Anti-CD90 & Mouse lgGI & $\begin{array}{l}\text { Human, rhesus, cynomolgus, } \\
\text { baboon, dog, pig }\end{array}$ & FITC & BD Pharmingen, 555595 \\
Anti-CDI05 & Mouse lgGI & Human & PErCP & BD Pharmingen, 560819 \\
\hline
\end{tabular}

Abbreviations: PE, phycoerythrin; FITC, fluorescein-5-isothiocyanate; PErCP, peridinin-chlorophyll-protein. 
San Jose, CA, USA). Resulting graphics were evaluated by Flowing Software (Turku Center for Biotechnology, University of Turku, Turku, Finland).

\section{Differentiation}

Osteogenic differentiation was tested in 70\% confluent, second to fourth passage rASCs and hASCs by adding $10^{-8}$ $\mathrm{M}$ dexamethasone, $5 \mu \mathrm{g} / \mathrm{mL}$ ascorbic acid (Sigma-Aldrich Co.), and $3.15 \mathrm{mg} / \mathrm{mL} \beta$-glycerophosphate (Sigma-Aldrich Co.) in the complete culture medium. Cells were maintained at $37^{\circ} \mathrm{C}$ and $5 \% \mathrm{CO}_{2}$, changing the medium every $3-4$ days. After 28 days, cells were fixed with paraformaldehyde 4\% and stained with Alizarin Red (Sigma-Aldrich Co.).

Adipogenic differentiation was tested in $100 \%$ confluent cells by adding $10^{-2} \mathrm{M}$ dexamethasone (Sigma-Aldrich Co.), $100 \mu \mathrm{M}$ indomethacin (Sigma-Aldrich Co.), $2.5 \mu \mathrm{g} / \mathrm{mL}$ insulin (Sigma-Aldrich Co.), and $0.5 \mathrm{mM}$ isobutilmetilxantin (Sigma-Aldrich Co.) in the complete culture medium. After 21 days, cells were fixed and then stained with Oil Red O (Sigma-Aldrich Co.).

The chondrogenic differentiation was tested using StemXVivo Human/Mouse Kit (R\&D Systems, Inc., Minneapolis, MN, USA) according to the manufacturer's protocol. Briefly, $2.5 \times 10^{5}$ cells were maintained as a pellet in a $15 \mathrm{~mL}$ conic tube under special medium. The medium was changed every 3 days until 30 days, when the chondrogenic pellets were fixed, histologically processed, and $5 \mu \mathrm{m}$ sections were stained with Alcian Blue (Sigma-Aldrich Co.).

\section{Colony forming units (CFUs)}

The CFU assay was performed by plating $5 \times 10^{2}$ second passage rASC and hASC cells in six-well culture plates for 5 days. The culture medium was removed, and plates were washed with PBS and incubated in 4\% paraformaldehyde for 30 minutes. Then, plates were washed with PBS and stained with Toluidine Blue 1\% (Sigma-Aldrich Co.) to count the number of colonies formed and the number of cells per colony. Results were analyzed by GraphPad Prism software (La Jolla, CA, USA).

\section{Growth curve and doubling time}

To determine the growth curve, six-well culture plates were plated with $1 \times 10^{4} \mathrm{rASC}$ and hASC first to fourth passage cells per well, counting a well every 24 hours from days 3 to 8 . The population doubling time was determined by the initial and final cell counting over time, according to the algorithm provided by Roth. ${ }^{19}$ Results were analyzed by GraphPad Prism software.

\section{Cytokinesis-block micronucleous assay}

To compare the nuclear stability of rASCs and hASCs, the cytokinesis-block micronucleus assay was performed as previously described by Fenech. ${ }^{20}$ Cells in passages 4-8 were plated in $25 \mathrm{~cm}^{2}$ culture bottles until they reached $80 \%$ of confluence when $5 \mathrm{mg} / \mathrm{mL}$ of cytochalasin-B (Sigma Aldrich) was added for 48 hours. Then, cells were dissociated, centrifuged, and treated for 3 minutes in a hypotonic solution composed of $0.075 \%$ potassium chlorite and $1 \%$ of complete medium in distilled water. Cells were fixed with $90 \%$ methanol and $10 \%$ acetic acid for 24 hours and observed using a microscope, where they were stained by Giemsa $0.5 \%$. A total of 600 binucleated cells of three biological samples of rASCs and hASCs were counted and analyzed using GraphPad Prism software.

\section{Statistical analyses}

Statistical significance was evaluated by Student's $t$-test for comparisons between rASCs and hASCs or two-way ANOVA followed by Bonferroni test when multiple times were considered, using GraphPad Prism software. All experiments were performed with at least three independent biological replicates (three donors). Differences between mean values were considered as significant when $P$ was $<0.05$.

\section{Results \\ Stemness characterization}

Flow cytometry analysis showed that rASCs and hASCs were both absent of hematopoietic markers CD34 and CD45 ( $<5 \%$ of expression) and positive to CD105 (both 99.9\%), CD73 (54\% vs 100\%), and CD90 (48\% vs 99.5\%) (Figure 1A). The expression of CD73 and CD90 was significantly lower in rabbit cells in comparison with that of human cells.

Differentiation assays showed that both rASCs and hASCs were able to generate osteocytes, adipocytes, and chondrocytes (Figure 1B). The differentiations were qualitatively verified by the staining of calcified extracellular matrix by Alizarin Red (osteogenic differentiation), lipid inclusions by Oil Red (adipogenic differentiation), and collagen fibers by Alcian Blue (chondrogenic differentiation).

\section{Proliferative profile}

The rASCs presented threefold higher potential to form fibroblastic colonies in vitro in comparison with hASCs. By the CFU assay, $74.7 \% \pm 9.9$ SD of plated rASCs were able to generate colonies with five cells or more, while only $23.2 \% \pm 1.1$ $\mathrm{SD}$ of plated hASCs resulted in colonies (Figure $2 \mathrm{~A}$ ). The 
A
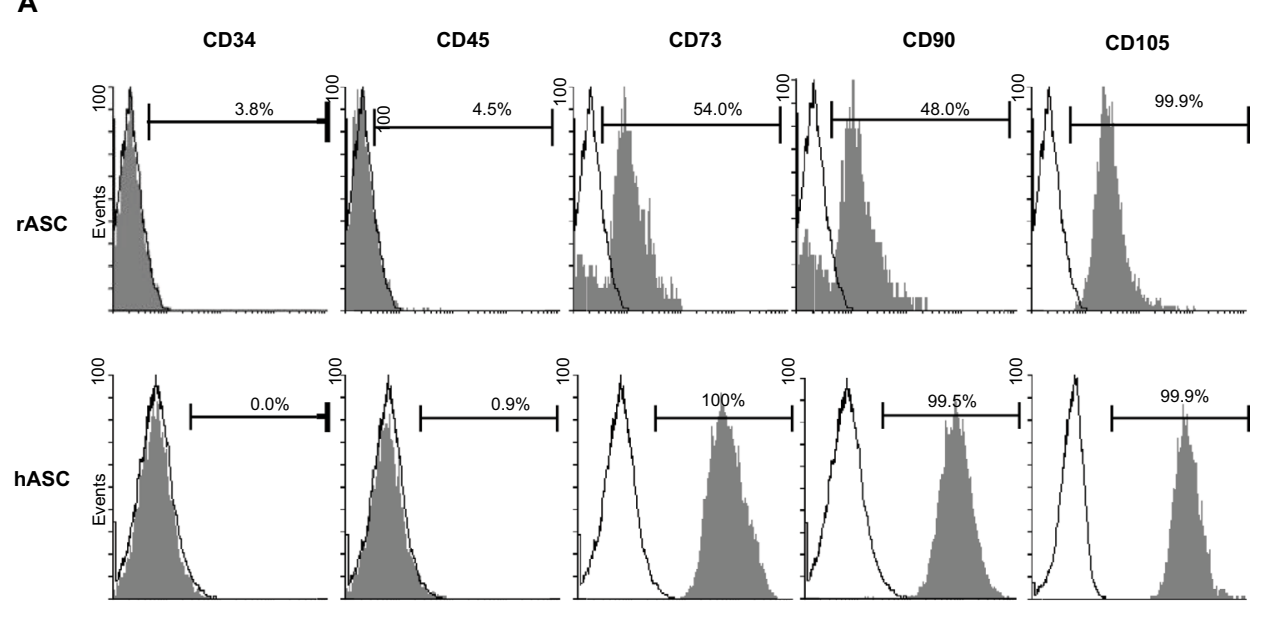

B

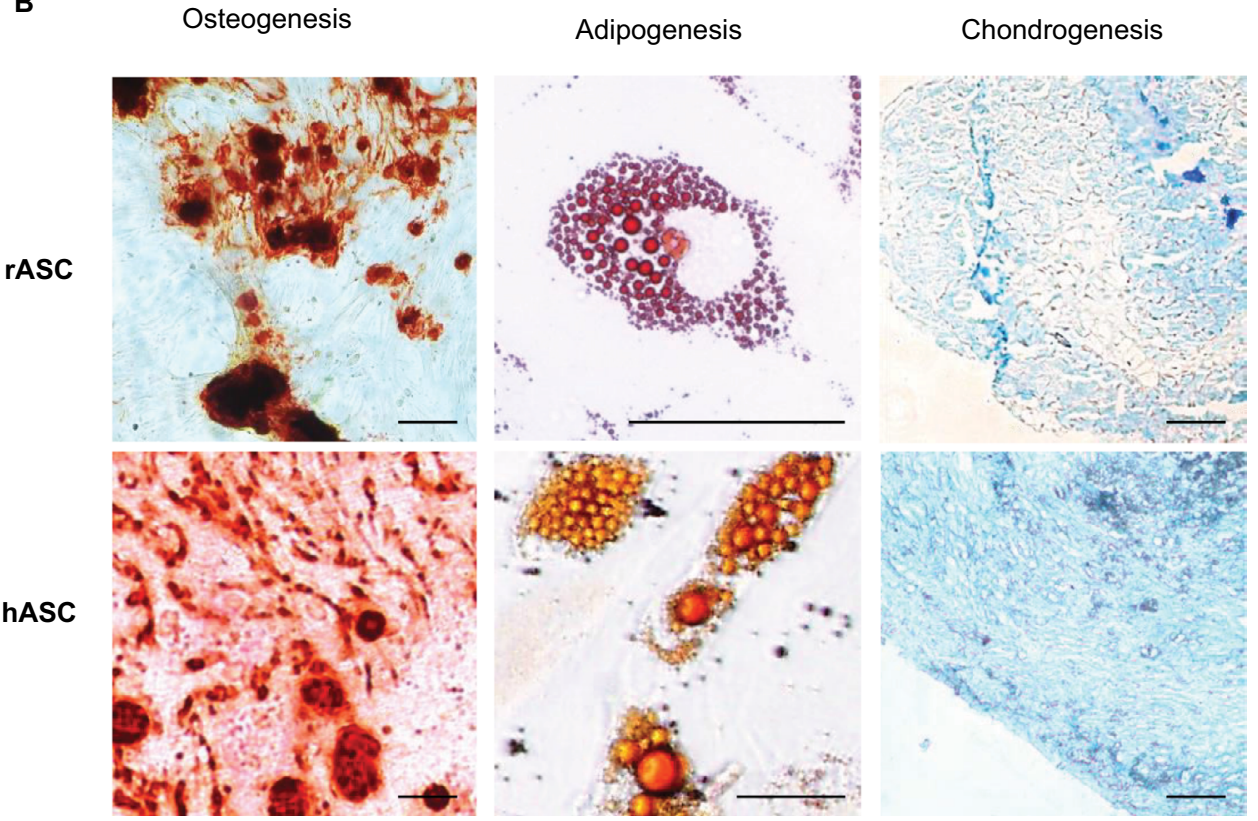

Figure I Stemness characterization.

Notes: (A) Representative graphics of flow cytometry analysis of immunophenotypic markers in rASCs and hASCs. White peaks represent control cells; gray peaks represent immunolabeled cells; bars represent the mean of positive expression from three biological replicates. (B) Differentiation potential of rASCs and hASCs for osteogenesis, adipogenesis, and chondrogenesis. Calcified extracellular matrix was detected by Alizarin Red; lipid inclusions were stained by Oil Red and collagen fibers by Alcian Blue. Bars: $50 \mu \mathrm{m}$.

Abbreviations: rASC, rabbit ASC; hASC, human ASC; ASC, adipose-derived MSC; MSC, mesenchymal stem cell.

sizes of the colonies were also significantly higher in rabbits, varying up to 73 cells in rASCs (mean of $21.3 \pm 15.4 \mathrm{SD}$ ) and up to 50 cells in hASCs (mean of 12.38 $\pm 7.6 \mathrm{SD}$; Figure 2B).

The growth curve assay showed a greater proliferation rate of rASCs than that of hASCs over time in culture (Figure 2C). The rASCs reached $100 \%$ of confluence after 8 days, increasing their population 24.8 times, while the hASC population grew 17 times. Accordingly, hASCs needed significantly more days to double their population $(2.3 \pm 0.17$ $\mathrm{SD}$ ) than rASCs (1.2 $\pm 0.17 \mathrm{SD}$; Figure 2D). Together, these findings demonstrate that rabbit cells feature higher clonogenic potential and proliferation capacity than human cells.

\section{Nuclear stability}

After we find that rASCs have a greater proliferative potential in vitro than the hASCs, we analyzed if their distinct proliferation rates and the culture conditions could cause nuclear alterations secondary to mitosis errors. Using the cytokinesis-block micronucleus assay, we observed nuclear buds (markers of gene amplification and/or elimination of 
A

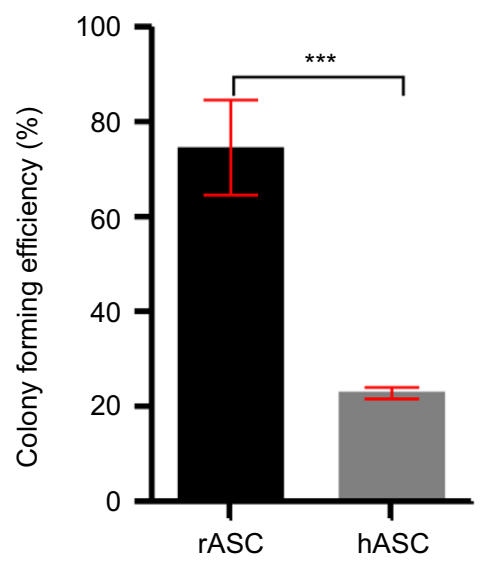

B

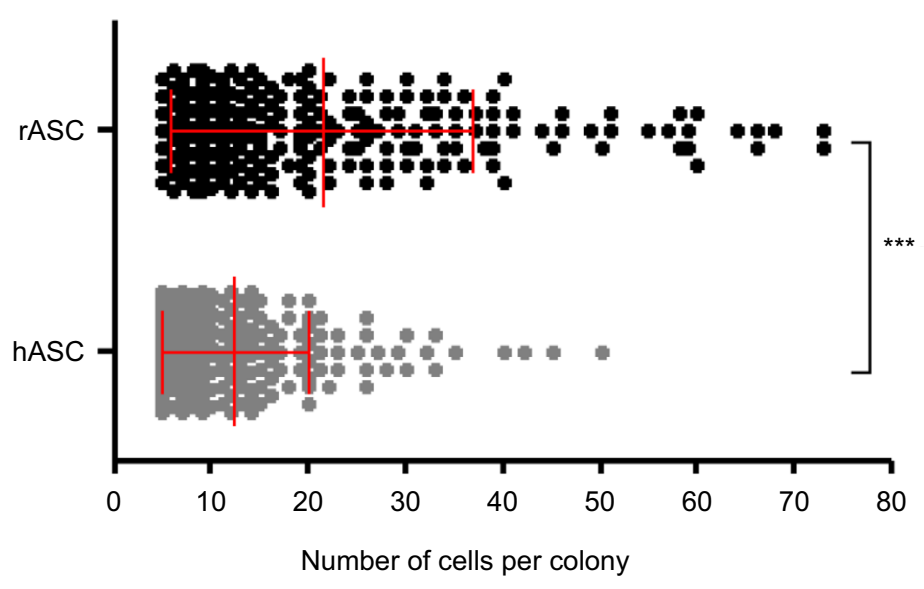

C

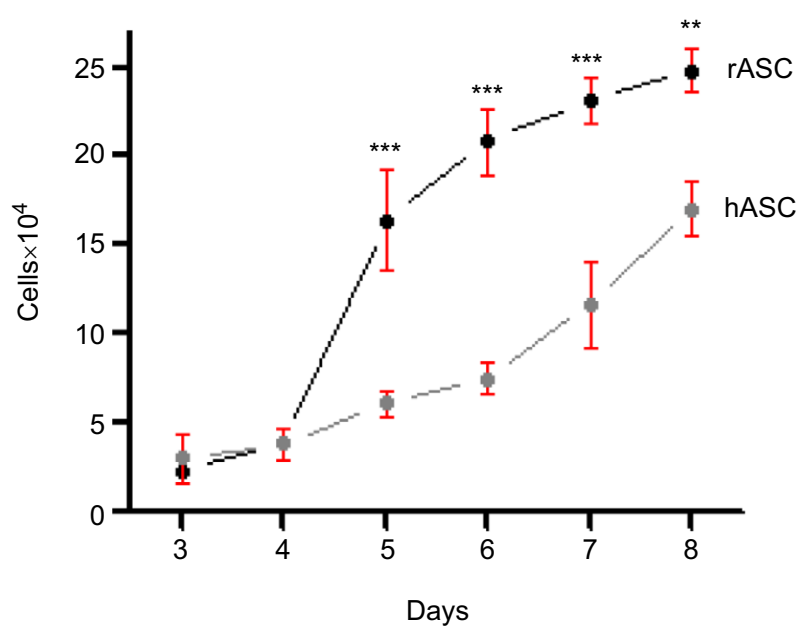

D

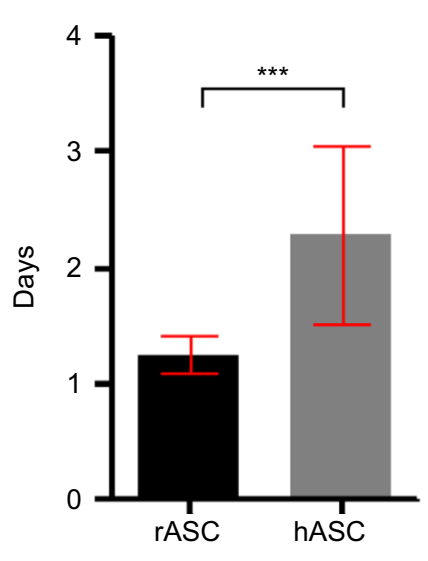

Figure 2 Proliferative profile of rASCs vs hASCs.

Notes: (A) Efficiency of CFUs. Bar reflects the mean of three independent biological replicates $\pm S D$. $* * * P<0.001$ using Student's $t$-test. (B) Number of cells per colony. Each dot represents one colony counted in three biological replicates. Lines show mean \pm SD. $* * * P<0.00 \mathrm{I}$ using Student's $t$-test. (C) Comparative growth curve of rASCs and hASCs, represented as number of cells over time. Each time point represents the mean of three biological replicates $\pm S D$. $* * * P<0.00 I$ and $* * P<0.0$ I by two-way ANOVA followed by the Bonferroni test. (D) Population doubling time. Bar reflects the mean of three biological replicates $\pm S D$. $* * * P<0.001$ using Student's $t$-test. Together, these results demonstrate the higher clonogenic and proliferative profile in vitro of rASCs in comparison to hASCs.

Abbreviations: rASC, rabbit ASC; hASC, human ASC; CFU, colony forming unit; ASC, adipose-derived MSC; MSC, mesenchymal stem cell.

DNA repair complexes), nucleoplasmic bridges (markers of DNA strand break misrepair and/or telomere end-fusions), and micronucleus (markers of chromosome breakage and/ or loss) in binucleated cells (Figure 3). ${ }^{20,21}$ In both rASCs and hASCs, we found low rates of nuclear alterations, with a relative frequency $<0.05$ of each type and no significant differences between the species. Thus, this result suggests that besides being highly proliferative, rASCs maintain their nuclear stability in vitro.

\section{Discussion}

MSCs derived from adipose tissue of different species have been widely studied in vitro and in vivo for various applications. $5,6,16,18$
The concept of MSCs as a heterogenic population and their distinct characteristics and potentials depending on sources and species has also been widely discussed., ${ }^{9,17,22,23}$ However, specific information regarding their distinct features and the implications for biotechnology applications and translational research is still scarce. In this study, we compared rASCs with hASCs and found that despite sharing similar differentiation potential and low rate of nuclear alterations over time in culture, they present distinct immunophenotype and proliferative profile. Interestingly, these findings could positively or negatively affect their use for different applications.

First, we showed that hASCs express significantly more CD73 and CD90 than rASCs. These markers are largely 
A

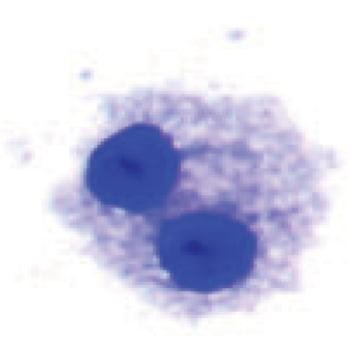

Binucleated cell

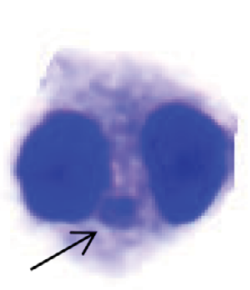

Micronucleus

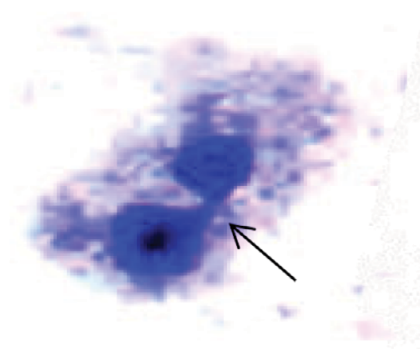

Nuclear bridge

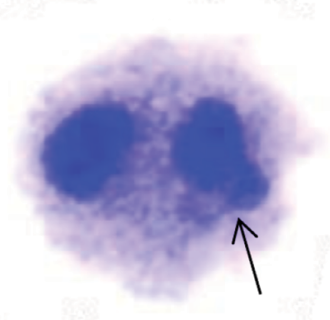

Nuclear bud

B
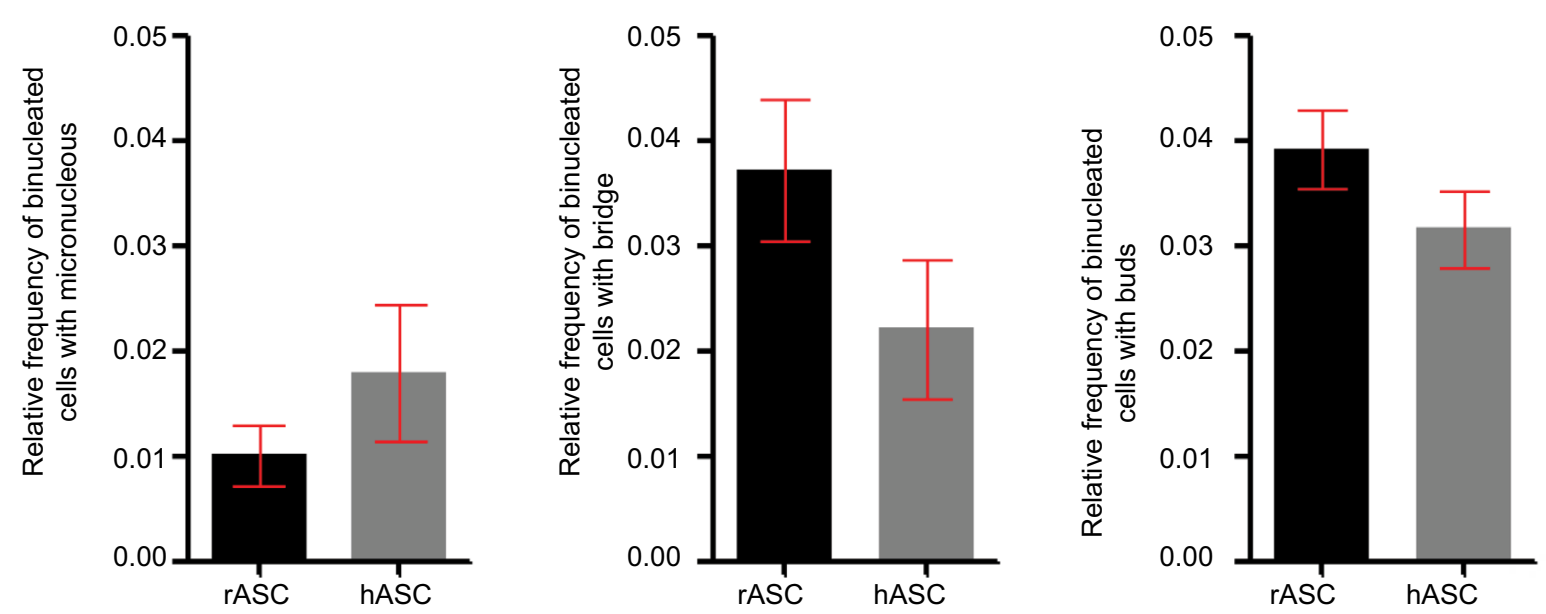

Figure 3 Nuclear stability.

Notes: (A) Normal binucleated cell and nuclear alterations observed by cytokinesis-block micronucleus assay. (B) Relative frequency of binucleated cells with nuclear alterations in rASCs and hASCs. Both rabbit and human cells present lower than 0.05 relative frequency of each error type, with no statistical relevance between species using Student's $t$-test.

Abbreviations: rASC, rabbit ASC; hASC, human ASC; ASC, adipose-derived MSC; MSC, mesenchymal stem cell.

known to be positive in MSCs from different sources, such as bone marrow, adipose tissue, dermis, muscle and umbilical cord blood, and from different species, such as humans, cats, dogs, and pigs. ${ }^{824-26}$ However, contradictory results have been described in rabbits, as summarized in Table 2. Martínez-Lorenzo et $\mathrm{al}^{18}$ found $40.5 \%$ of CD90 expression in rASCs and negative expression of CD73 and CD105. In contrast, Sunay et $\mathrm{al}^{5}$ showed $99 \%$ of CD73-positive cells. In addition, the adipose tissue is not the only source of rabbit MSCs with distinct immunophenotype. Bakhtina et $\mathrm{al}^{27}$ found absence of CD90 expression, and Lee et $\mathrm{al}^{28}$ observed no expression of CD73, CD90, and CD105 in rabbit bone marrow-derived MSCs. Despite the tested markers, other authors found that both rabbit and human MSCs are positive to CD29, CD44, and Sox2 while negative to CD14. ${ }^{11,27-29}$ Furthermore, the disparities in the results could be due to unspecific antibodies, as anti-rabbit antibodies are not widely available and most studies were performed using reagents with no described reaction against rabbits. Differences in the passage of cells used in each study and variations in the methodologies of rASC isolation or immunostaining could also affect the results. ${ }^{26}$

Despite the immunophenotyping of MSCs having been used widely in different forms to characterize cells, the biological significance of these findings is not commonly discussed. Both CD73 and CD90 act as signaling molecules to various cell-cell and cell-matrix interactions. ${ }^{30,31}$ The CD73 or ecto-5 $\phi$-nucleotidase plays a role in physiological responses such as epithelial ion and fluid transport, ischemic preconditioning, tissue injury, platelet function, hypoxia, vascular leak, drug resistance, and tumor promotion. ${ }^{31}$ In addition, immunosuppressive effects via CD73 mechanisms are described in MSCs..$^{32}$ For example, a study using rat CD73+ MSCs suggests that this protein exerts a powerful anti-inflammatory effect 
Table 2 Distinct findings of immunophenotypic markers' expression in rabbit MSC studies

\begin{tabular}{|c|c|c|c|c|}
\hline Reference & Source & CD73 (\%) & CD90 (\%) & CDIO5 (\%) \\
\hline This study & Adipose tissue & 54 & 48 & 99.9 \\
\hline Martínez-Lorenzo et al ${ }^{18}$ & Adipose tissue & 1.6 & 40.1 & 20.5 \\
\hline Sunay et $\mathrm{al}^{5}$ & Adipose tissue & 99 & - & 99 \\
\hline Bakhtina et $\mathrm{al}^{27}$ & Bone marrow & - & 0.1 & - \\
\hline Lee et $\mathrm{al}^{28}$ & Bone marrow & 0.5 & 1.2 & 1.6 \\
\hline Tan et $\mathrm{al}^{54}$ & Bone marrow & 96.4 & 96.9 & - \\
\hline Kovac et $\mathrm{al}^{55}$ & Amniotic fluid & 1.2 & 17.1 & 2.5 \\
\hline
\end{tabular}

Note: Values represent the mean described by the authors; - denotes not evaluated.

Abbreviation: MSC, mesenchymal stem cell.

critical for cardiac recovery following myocardial ischemia/ reperfusion injury. ${ }^{33}$ It is also suggested that CD73 expression is related to MSC migratory potential and direct bone repair and regeneration. ${ }^{34,35}$ Similarly, the CD90 or Thy- 1 is described to play a role in the synthesis and releasing of growth factors, cytokines, and extracellular matrix components to assist tissue repair during inflammation and wound healing. ${ }^{30} \mathrm{CD} 90$ is also reported to affect stem cells' growth, differentiation, and immunosuppression. ${ }^{36-38}$ Thus, the distinct expression of CD73 and CD90 in rabbit MSCs could be implicated in failure to translate results obtained in the rabbit model to clinic. Nevertheless, care should be taken when interpreting animal findings to human uses.

Despite presenting distinct immunophenotype, rASCs and hASCs were both able to generate several mesodermal tissues, demonstrating that their differentiation potential was not affected. Similar qualitative results were previously described with no differences between rabbit and human cells. ${ }^{5,27}$ However, our study could not infer quantitative or temporal information of rASC and hASC differentiation potential. Several independent quantitative studies showed contrasting results, while Bakhtina et al found a higher differentiation potential of human bone morrow-derived MSCs for chondrocytes, osteocytes, and adipocytes. ${ }^{27}$ MartínezLorenzo et al ${ }^{18}$ suggested a lower chondrogenic potential of hASCs in comparison with rabbit cells. As the MSCs are credited to their differentiation potential and paracrine effects, it would be useful to elucidate if differentiation potential of human and rabbit MSCs is distinct in some level.

After characterizing stemness features, we compared rabbit and human MSC proliferative profiles, where rASCs presented greater clonogenic potential and proliferation rate than hASCs. However, a previous study detected a higher clonogenic potential of rASCs but no difference in their growth curve. ${ }^{18}$ Anyway, the remarkable proliferation of rASCs was previously reported in various studies when comparing the rabbit model with other animal models or the rASCs with other sources of MSCs. ${ }^{17,37,39}$ Interestingly, the proliferative rate of MSCs differently affects their use in preclinical studies and biotechnology applications.

On one side, the possibility of achieving a great number of cells in a short period of time is desired for cell-based therapy studies. Preclinical protocols testing MSC transplantation or their association in tissue engineering often require millions to billions of cells, so the high proliferative rate of rASCs would be useful. ${ }^{40}$ For example, preclinical studies used rabbit MSCs in the treatment of osteochondral defects, cardiovascular diseases, and skin wound healing with great outcomes. ${ }^{41-43}$ In addition, the rabbit is the elected model for pharmacokinetic studies due its strong correlation with clinical trials. ${ }^{44}$ For instance, the rabbit ear scar model was successfully used prior to a Phase I randomized clinical trial for safety and tolerability of topically delivered kynurenic acid in humans and randomized controlled trials. ${ }^{45}$ Thus, the rASC fast proliferation consists of an advantageous characteristic, promoting vast MSC availability for both in vitro and in vivo applications with a great translation described to human clinical medicine.

On the other hand, biotechnology studies have suggested that fast proliferation can be detrimental to cell reprogramming. . $^{39,46}$ For instance, Honda et $\mathrm{al}^{39}$ described not been able to induce pluripotency in rabbit fibroblasts due to their high proliferation rate. Similarly, our group could not effectively reprogram rASCs using the same protocols successfully applied in other species, such as dog, horse, bovine, and human. ${ }^{47-50}$ The induced pluripotent stem cell (iPS)-like colonies vanished after few days, possibly due to the high proliferation of rASCs (Figure S1A and B). After cell cycle arrest and synchronization by serum starvation, we were able to reduce the proliferative rate of rASCs and improve the transduction and reprogramming (Figure S1C-G). ${ }^{51}$ During mitosis, chromosomes were tightly condensed, making it difficult for transcription factors to bind to the DNA, which could explain why a high proliferative profile may impair cell transduction. ${ }^{52}$ However, the mechanisms 
Table 3 Summary of comparative characterization of rASCs and hASCs

\begin{tabular}{|c|c|c|c|}
\hline Feature & rASC & hASC & Implications \\
\hline Immunophenotype & 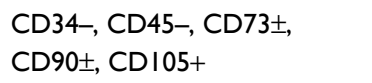 & $\begin{array}{l}\text { CD34-, CD45-, CD73+, } \\
\text { CD90+, CDI05+ }\end{array}$ & $\begin{array}{l}\text { MSC behavior and possible failure to } \\
\text { translation }\end{array}$ \\
\hline $\begin{array}{l}\text { Differentiation potential } \\
\text { (qualitative) }\end{array}$ & $\begin{array}{l}\text { Osteocytes, chondrocytes, and } \\
\text { adipocytes }\end{array}$ & $\begin{array}{l}\text { Osteocytes, chondrocytes, } \\
\text { and adipocytes }\end{array}$ & $\begin{array}{l}\text { Unclear due to contrasting quantitative } \\
\text { studies }\end{array}$ \\
\hline Proliferative profile & Higher & Lower & $\begin{array}{l}\text { Good source of cells to preclinical } \\
\text { therapeutic applications but detrimental to } \\
\text { reprogramming }\end{array}$ \\
\hline Nuclear alterations & Low & Low & Safe for cell therapy \\
\hline
\end{tabular}

Abbreviations: rASC, rabbit ASC; hASC, human ASC; MSC, mesenchymal stem cell; ASC, adipose-derived MSC.

regarding the influence of the proliferation in the reprogramming process are still poorly understood.

In addition, high proliferation and culture conditions are related to cell mutations. ${ }^{52,53}$ Malignant mutations can generate tumors in vivo, so low levels of errors are required for safe cell therapy applications. ${ }^{21}$ Interestingly, we found no significant difference between rabbit and human cells, and both presented low frequency of errors, similar to previously described in literature in human cells. ${ }^{20,21}$ For our knowledge, this is the first report of cytokinesis-block micronucleus assay in rabbit MSCs. This methodology is useful to predict risk factors; however, karyotyping or other genetic analysis is required to identify specific chromosomal alterations. ${ }^{21}$ Together, these findings suggest that despite having a high proliferation, rASCs maintain their nuclear stability in vitro and could be safely used in preclinical therapeutic studies.

This study highlighted the distinct features of rASCs and hASCs and their possible implications to biotechnology applications and translational studies (Table 3). Both ASCs presented differentiation potential to osteocytes, chondrocytes, and adipocytes and shared similar immunophenotype expression to CD105+, CD34-, and CD45-, but rabbit cells expressed significantly less CD73 and CD90 than human cells. In addition, rASCs presented greater clonogenic potential and proliferation rate than hASCs but no difference in nuclear alterations. Future assays regarding the differentiation potential of rASCs and hASCs would be useful to elucidate if there are quantitative interspecific variations. In addition, authors should carefully evaluate results in the rabbit model as some specific features could affect translation of animal findings to future human applications.

\section{Conclusion}

rASCs and hASCs present distinct features regarding their immunophenotype and proliferative profiles that can positively or negatively affect their use for different applications in biotechnology (such as cell reprogramming) and translational studies (such as cell transplantation, tissue engineering, and pharmacokinetics). Despite their differences, rabbit studies have shown great translation to clinic; thus, these particularities between rASCs and hASCs should not prevent their use in in vitro and preclinical models, but care should be taken to interpret results and properly translate animal findings to medicine.

\section{Abbreviations}

ASC, adipose-derived MSC; CFUs, colony forming units; FITC, fluorescein-5-isothiocyanate; hASC, human ASC; iPS, induced pluripotent stem cells; MSC, mesenchymal stem cell; PE, phycoerythrin; PErCP, peridinin-chlorophyll-protein; rASC, rabbit ASC.

\section{Acknowledgments}

This work was supported by the grants 2012/04196-04, 2013/09392-9, and 2017/21266-0, São Paulo Research Foundation (FAPESP). We are grateful to the technical support of Laboratory Multiuser for Biological Studies (LAMEB) of Santa Catarina Federal University and Dr Debora Afonso Cornélio for the micronucleus assay training.

\section{Disclosure}

Helena Zomer has received grants from Fundação de Amparo a Pesquisa do Estado de São Paulo, during the conduct of the study, and grants from Coordenação de Aperfeiçoamento de Pessoal de Nível Superior, outside the submitted work. The other authors report no conflicts of interest in this work.

\section{References}

1. Intawicha P, Ou YW, Lo NW, et al. Characterization of embryonic stem cell lines derived from New Zealand white rabbit embryos. Cloning Stem Cells. 2009;11(1):27-38.

2. Calasans-Maia MD, Monteiro ML, Ascoli FO, Granjeiro JM. The rabbit as an animal model for experimental surgery. Acta Cir Bras. 2009;24(4):325-328. 
3. Mapara M, Thomas BS, Bhat KM. Rabbit as an animal model for experimental research. Dent Res J. 2012;9(1):111-118.

4. Püschel B, Viebahn C. Rabbit mating and embryo isolation. Cold Spring Harb Protoc. 2010;2010(1):pdb.prot5350.

5. Sunay O, Can G, Cakir Z, et al. Autologous rabbit adipose tissue-derived mesenchymal stromal cells for the treatment of bone injuries with distraction osteogenesis. Cytotherapy. 2013;15(6):690-702.

6. Fekrazad R, Eslaminejad MB, Shayan AM, et al. Effects of Photobiomodulation and Mesenchymal Stem Cells on Articular Cartilage Defects in a Rabbit Model. Photomed Laser Surg. 2016;34(11):543-549.

7. Isakson M, de Blacam C, Whelan D, Mcardle A, Clover AJ. Mesenchymal Stem Cells and Cutaneous Wound Healing: Current Evidence and Future Potential. Stem Cells Int. 2015;2015:1-12.

8. Dominici M, Le Blanc K, Mueller I, et al. Minimal criteria for defining multipotent mesenchymal stromal cells. The International Society for Cellular Therapy position statement. Cytotherapy. 2006;8(4):315-317.

9. da Silva Meirelles L, Chagastelles PC, Nardi NB, Meirelles S. Mesenchymal stem cells reside in virtually all post-natal organs and tissues. J Cell Sci. 2006;119(Pt 11):2204-2213.

10. Sachs PC, Francis MP, Zhao M, et al. Defining essential stem cell characteristics in adipose-derived stromal cells extracted from distinct anatomical sites. Cell Tissue Res. 2012;349(2):505-515.

11. Bahn J, Chung J, Im W, Kim M, Kim SH. Science Suitability of autologous serum for expanding rabbit adipose-derived stem cell populations. 2012;13(5):413-417.

12. Cherubino M, Rubin JP, Miljkovic N, Kelmendi-Doko A, Marra KG. Adipose-derived stem cells for wound healing applications. Ann Plast Surg. 2011;66(2):210-215.

13. Du Y, Roh DS, Funderburgh ML, et al. Adipose-derived stem cells differentiate to keratocytes in vitro. Mol Vis. 2010;16(9):2680-2689.

14. Kim JH, Jung M, Kim HS, Kim YM, Choi EH. Adipose-derived stem cells as a new therapeutic modality for ageing skin. Exp Dermatol. 2011;20(5):383-387.

15. Falanga V, Iwamoto S, Chartier M, et al. Autologous bone marrowderived cultured mesenchymal stem cells delivered in a fibrin spray accelerate healing in murine and human cutaneous wounds. Tissue Eng. 2007;13(6):1299-1312.

16. Abudusaimi A, Aihemaitijiang Y, Wang YH, Cui L, Maimaitiming S, Abulikemu M. Adipose-derived stem cells enhance bone regeneration in vascular necrosis of the femoral head in the rabbit. $J$ Int Med Res. 2011;39(5):1852-1860.

17. Arrigoni E, Lopa S, de Girolamo L, Stanco D, Brini AT. Isolation, characterization and osteogenic differentiation of adipose-derived stem cells: from small to large animal models. Cell Tissue Res. 2009;338(3):401-411.

18. Martínez-Lorenzo MJ, Royo-Cañas M, Alegre-Aguarón E, et al. Phenotype and chondrogenic differentiation of mesenchymal cells from adipose tissue of different species. J Orthop Res. 2009;27(11):1499-1507.

19. Roth V [webpage on the Internet]. Doubling Time Computing. 2006. Available from: http://www.doubling-time.com/compute.php. Accessed April 8, 2018.

20. Fenech M. Cytokinesis-block micronucleus cytome assay. Nat Protoc. 2007;2(5):1084-1104

21. Cornélio DA, Tavares JC, Pimentel TV, Cavalcanti GB, Batistuzzo de Medeiros SR, Medeiros SRB. Cytokinesis-block micronucleus assay adapted for analyzing genomic instability of human mesenchymal stem cells. Stem Cells Dev. 2014;23(8):823-838.

22. Ozturk S, Karagoz H. Experimental stem cell therapies on burn wound: do source, dose, timing and method matter? Burns. 2015;41(6): 1133-1139.

23. Zomer HD, Vidane AS, Gonçalves NN, Ambrósio CE. Mesenchymal and induced pluripotent stem cells: general insights and clinical perspectives. Stem Cells Cloning. 2015;8(8):S263-34

24. Kisiel AH, Mcduffee LA, Masaoud E, Bailey TR, Esparza Gonzalez BP, Nino-Fong R. Isolation, characterization, and in vitro proliferation of canine mesenchymal stem cells derived from bone marrow, adipose tissue, muscle, and periosteum. Am J Vet Res. 2012;73(8): $1305-1317$.
25. Clark KC, Fierro FA, Ko EM, et al. Human and feline adipose-derived mesenchymal stem cells have comparable phenotype, immunomodulatory functions, and transcriptome. Stem Cell Res Ther. 2017;8(1):1-16.

26. Lee AY, Lee J, Kim CL, et al. Comparative studies on proliferation, molecular markers and differentiation potential of mesenchymal stem cells from various tissues (adipose, bone marrow, ear skin, abdominal skin, and lung) and maintenance of multipotency during serial passages in miniature pig. Res Vet Sci. 2015;100:115-124.

27. Bakhtina A, Tohfafarosh M, Lichtler A, Arinzeh TL. Characterization and differentiation potential of rabbit mesenchymal stem cells for translational regenerative medicine. In Vitro Cell Dev Biol Anim. 2014;50(3):251-260.

28. Lee TC, Lee TH, Huang YH, et al. Comparison of surface markers between human and rabbit mesenchymal stem cells. PLoS One. 2014;9(11):e111390.

29. Ercolin AC, Roballo KC, Casals JB, et al. Rabbit olfactory stem cells. Isolation protocol and characterization. Acta Cir Bras. 2016;31(1):59-66.

30. Kisselbach L, Merges M, Bossie A, Boyd A. CD90 Expression on human primary cells and elimination of contaminating fibroblasts from cell cultures. Cytotechnology. 2009;59(1):31-44.

31. Zhang B. CD73: a novel target for cancer immunotherapy. Cancer Res. 2010;70(16):6407-6411.

32. Kerkelä E, Laitinen A, Räbinä J, et al. Adenosinergic Immunosuppression by Human Mesenchymal Stromal Cells Requires Co-Operation with T cells. Stem Cells. 2016;34(3):781-790.

33. Shin EY, Wang L, Zemskova M, et al. Adenosine production by biomaterial-supported mesenchymal stromal cells reduces the innate inflammatory response in myocardial ischemia/reperfusion injury. $\mathrm{J} \mathrm{Am}$ Heart Assoc. 2018;7(2):e006949.

34. Ode A, Kopf J, Kurtz A, et al. CD73 and CD29 concurrently mediate the mechanically induced decrease of migratory capacity of mesenchymal stromal cells. Eur Cell Mater. 2011;22:26-42.

35. Ode A, Schoon J, Kurtz A, et al. CD73/5'-ecto-nucleotidase acts as a regulatory factor in osteo-/chondrogenic differentiation of mechanically stimulated mesenchymal stromal cells. European Cells and Materials. 2012;25:37-47.

36. Campioni D, Rizzo R, Stignani M, et al. A decreased positivity for CD90 on human mesenchymal stromal cells (MSCs) is associated with a loss of immunosuppressive activity by MSCs. Cytometry B Clin Cytom. 2009;76(3):225-230.

37. Moraes DA, Sibov TT, Pavon LF, et al. A reduction in CD90 (THY-1) expression results in increased differentiation of mesenchymal stromal cells. Stem Cell Res Ther. 2016;7(1):1-14.

38. Peptan IA, Hong L, Mao JJ. Comparison of osteogenic potentials of visceral and subcutaneous adipose-derived cells of rabbits. Plast Reconstr Surg. 2006;117(5):1462-1470.

39. Honda A, Hirose M, Hatori M, et al. Generation of induced pluripotent stem cells in rabbits: potential experimental models for human regenerative medicine. J Biol Chem. 2010;285(41):31362-31369.

40. Gómez-Barrena E, Padilla-Eguiluz NG, Avendaño-Solá C, et al. A multicentric, open-label, randomized, comparative clinical trial of two different doses of expanded hbm-mscs plus biomaterial versus iliac crest autograft, for bone healing in nonunions after long bone fractures: Study Protocol. Stem Cells Int. 2018;2018:1-13.

41. Zhang H, Li ZL, Su XZ, et al. Subchondral bone derived mesenchymal stem cells display enhanced osteo-chondrogenic differentiation, selfrenewal and proliferation potentials. Exp Anim. 2018;67(3):349-359.

42. Pelizzo G, Avanzini MA, Icaro Cornaglia A, et al. Mesenchymal stromal cells for cutaneous wound healing in a rabbit model: preclinical study applicable in the pediatric surgical setting. J Transl Med. 2015;13(1):219

43. Nesselmann C, Ma N, Bieback K, et al. Mesenchymal stem cells and cardiac repair. J Cell Mol Med. 2008;12(5B):1795-1810.

44. Rifat D, Prideaux B, Savic RM, et al. Pharmacokinetics of rifapentine and rifampin in a rabbit model of tuberculosis and correlation with clinical trial data. Sci Transl Med. 2018;10(435):eaai7786.

45. Papp A, Hartwell R, Evans M, Ghahary A. The Safety and Tolerability of Topically Delivered Kynurenic Acid in Humans. A Phase 1 Randomized Double-Blind Clinical Trial. J Pharm Sci. 2018;107(6):1572-1576. 
46. Xu Y, Wei X, Wang M, et al. Proliferation rate of somatic cells affects reprogramming efficiency. J Biol Chem. 2013;288(14):9767-9778.

47. Gonçalves NJN, Bressan FF, Roballo KCS, et al. Generation of LIFindependent induced pluripotent stem cells from canine fetal fibroblasts. Theriogenology. 2017;92(1):75-82.

48. Gustali M, Bressa FF, Maziero R, et al. Induced pluripotent stem cells (iPS) derived from equine umbilical cord cells using lentivirus vector stemcca. Reprod Fertil Dev. 2014;26(1):213.

49. Bressan FF, Therrien J, Filion F, Perecin F, Smith LC, Meirelles FV. Abnormal dna methylation patterns and allele-specific expression of imprinted genes in bovine-induced pluripotent stem cells. Reprod Fertil Dev. 2015;27(1):254.

50. Rochetti R, Bressan FF, Regiani T, Labate CA, Fraietta R, Meirelles FV. Comparative analysis of the lipid profile of human mesenchymal stem cells induced to pluripotency by different transfection factors. Fertil Steril. 2013;100(3):S456-S457.
51. Chen M, Huang J, Yang X, et al. Serum starvation induced cell cycle synchronization facilitates human somatic cells reprogramming. PLoS One. 2012;7(4):e28203.

52. Alberts B, Johnson AD, Lewis J, Morgan D, Raff M, Roberts K, Walter P. Molecular Biology of the Cell. 6th ed. New York: W. W. Norton \& Company; 2014.

53. Kim M, Rhee JK, Choi H, et al. Passage-dependent accumulation of somatic mutations in mesenchymal stromal cells during in vitro culture revealed by whole genome sequencing. Sci Rep. 2017;7(1):14508.

54. Tan SL, Ahmad TS, Selvaratnam L, Kamarul T. Isolation, characterization and the multi-lineage differentiation potential of rabbit bone marrow-derived mesenchymal stem cells. J Anat. 2013;222(4):437-450.

55. Kovac M, Vasicek J, Kulikova B, et al. Different RNA and protein expression of surface markers in rabbit amniotic fluid-derived mesenchymal stem cells. Biotechnol Prog. 2017;33(6):1601-1613. 


\section{Supplementary material}

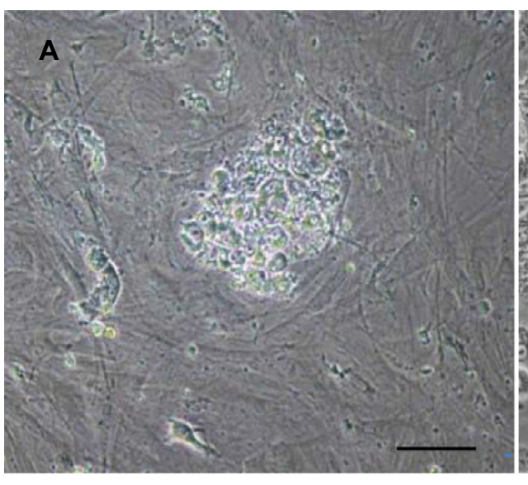

D
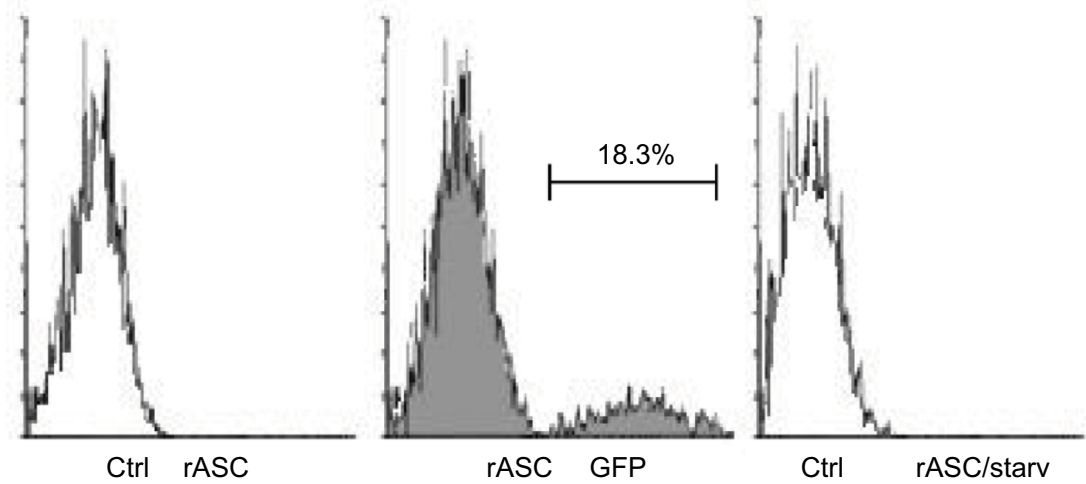

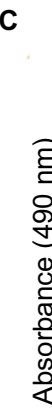
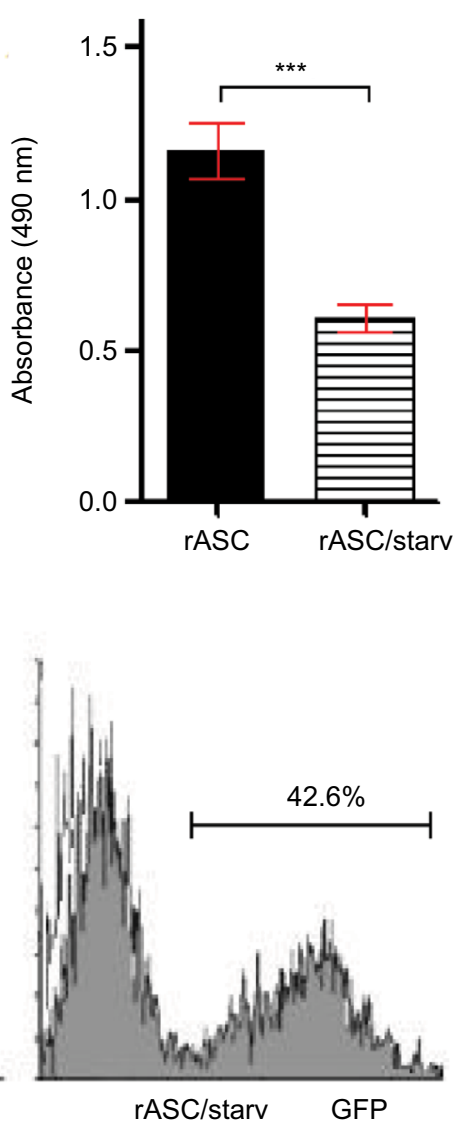
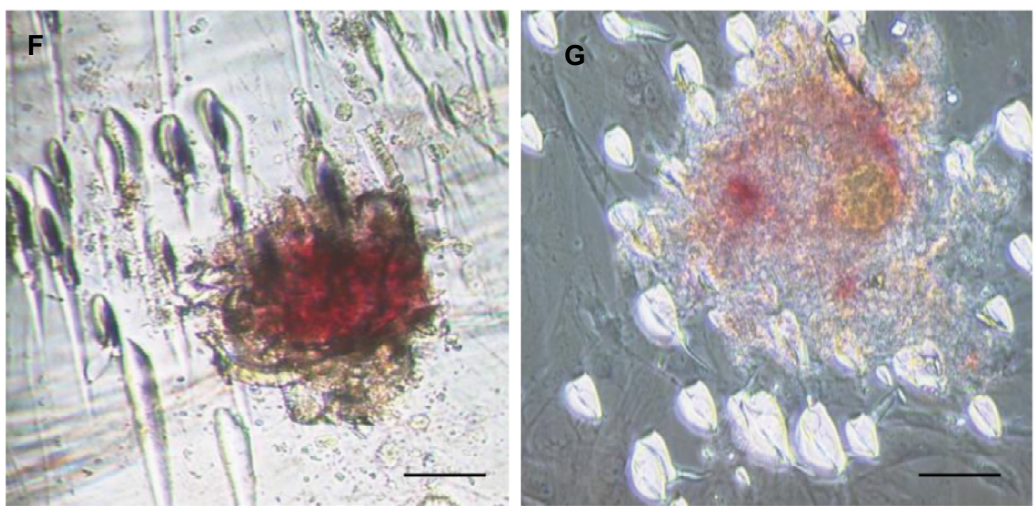

Figure SI Induction of pluripotency in rASCs.

Notes: rASCs after pluripotency induction showing iPS initial characteristic morphology (A) and partial reprogramming morphology (B). All colonies vanished after few days, possibly due to the high proliferation of rASCs. (C) Serum starvation treatment ( $r$ ASC/starv) successfully decreased rASC activity, as shown by MTS viability assay $(* * * P<0.00 \mathrm{I}$ using Student's $t$-test). In the same way, starved cells transduced with a lentivirus carrying the GFP plasmid presented a higher expression than not starved rASCs by flow cytometry analysis (D), suggesting that the high proliferation of rASCs is detrimental to reprogramming and controlling the cell cycle by starvation can improve the pluripotency induction. Starved rASC-derived iPS colonies showing characteristic morphology $(\mathbf{E})$ and positive alkaline phosphate staining under bright field $(\mathbf{F})$ and phase contrast microscopy (G). Bars: $50 \mu \mathrm{m}$.

Abbreviations: rASC; rabbit ASC; iPS, induced pluripotent stem cell; Ctrl, control; ASC, adipose-derived MSC; MSC, mesenchymal stem cell; MTS, 3-(4,5-dimethylthiazol2-yl)-5-(3-carboxymethoxyphenyl)-2-(4-sulfophenyl)-2H-tetrazolium). 


\section{Publish your work in this journal}

Stem Cells and Cloning: Advances and Applications is an international, peer-reviewed, open access journal. Areas of interest in stem cell research include: Embryonic cell stems; Adult stem cells; Blastocysts; Cordblood stem cells; Stem cell transformation and culture; Therapeutic cloning; Umbilical cord blood and bone marrow cells; Laboratory, animal and human therapeutic studies; Philosophical and ethical issues related to stem cell research. This journal is indexed on CAS. The manuscript management system is completely online and includes a quick and fair peer-review system. Visit http://www.dovepress.com/ testimonials.php to read real quotes from published authors..

Submit your manuscript here: https://www.dovepress.com/stem-cells-and-cloning-advances-and-applications-journal 